\title{
The effect of Molecular Structure on Stability of Organic Nanoparticles formed by Bodipy Dimers
}

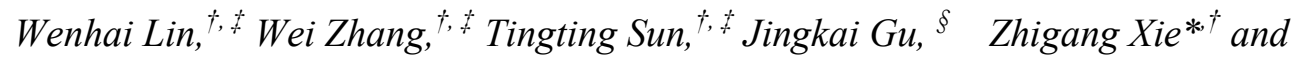
Xiabin Jing ${ }^{\dagger}$

${ }^{\dagger}$ State Key Laboratory of Polymer Physics and Chemistry, Changchun Institute of Applied Chemistry, Chinese Academy of Sciences, 5625 Renmin Street, Changchun, Jilin 130022, P. R. China

${ }^{\ddagger}$ University of Chinese Academy of Sciences, Beijing 100049, P. R. China

${ }^{\S}$ Research Center for Drug Metabolism, College of Life Sciences, Jilin University, Changchun 130012, P. R. China

\section{Corresponding Author}

*Zhigang Xie

E-mail: xiez@ciac.ac.cn 


\section{Content}

1. Figure S1. MALDI-TOF-MS spectra of the SNBDP.

2. Figure S2. (A) ${ }^{1} \mathrm{H}$ NMR characterization and (B) MALDI-TOF MS spectrum of C4NBDP.

3. Figure S3. (A) ${ }^{1} \mathrm{H}$ NMR characterization and (B) MALDI-TOF MS spectrum of C6NBDP.

4. Figure S4. (A) ${ }^{1} \mathrm{H}$ NMR characterization and (B) MALDI-TOF MS spectrum of C9NBDP.

5. Figure S5. FT-IR spectra of C8NBDP NPs and C9NBDP NPs freeze-dried.

6. Figure S6. The diameter and PdI of (A) C4NBDP NPs, (B) C6NBDP NPs, (C) C8NBDP NPs and (D) C9NBDP NPs in twenty days measured by DLS.

7. Figure S7. The photos of CxNBDP NPs in water and CxNBDP in $\mathrm{MeOH}$ under 365-nm light irradiation.

8. Figure S8. Cell viability of HeLa cells incubated with CxNBDP NPs.

9. Figure S9. Respresentative CLSM images of HeLa cells incubated with C6NBDP NPs for $1 \mathrm{~h}$ and $3 \mathrm{~h}$.

10. Figure S10. Representative CLSM images of HeLa cells incubated with C8NBDP NPs for $1 \mathrm{~h}$ and $3 \mathrm{~h}$.

11. Figure S11. (A) UV-vis absorption and (B) fluorescence spectra of Nile red@C9NBDP NPs.

12. Table S1. Zeta potential of CxNBDP NPs 


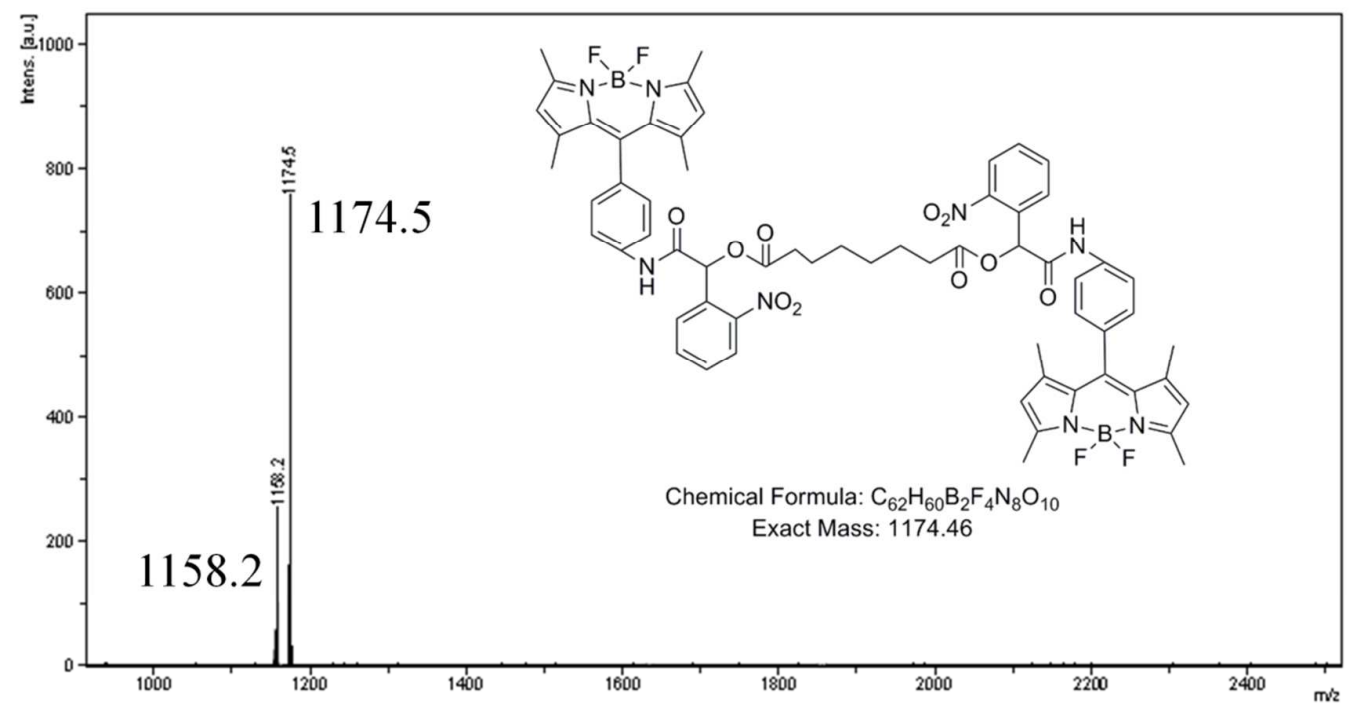

Figure S1. MALDI-TOF-MS spectra of the SNBDP. 

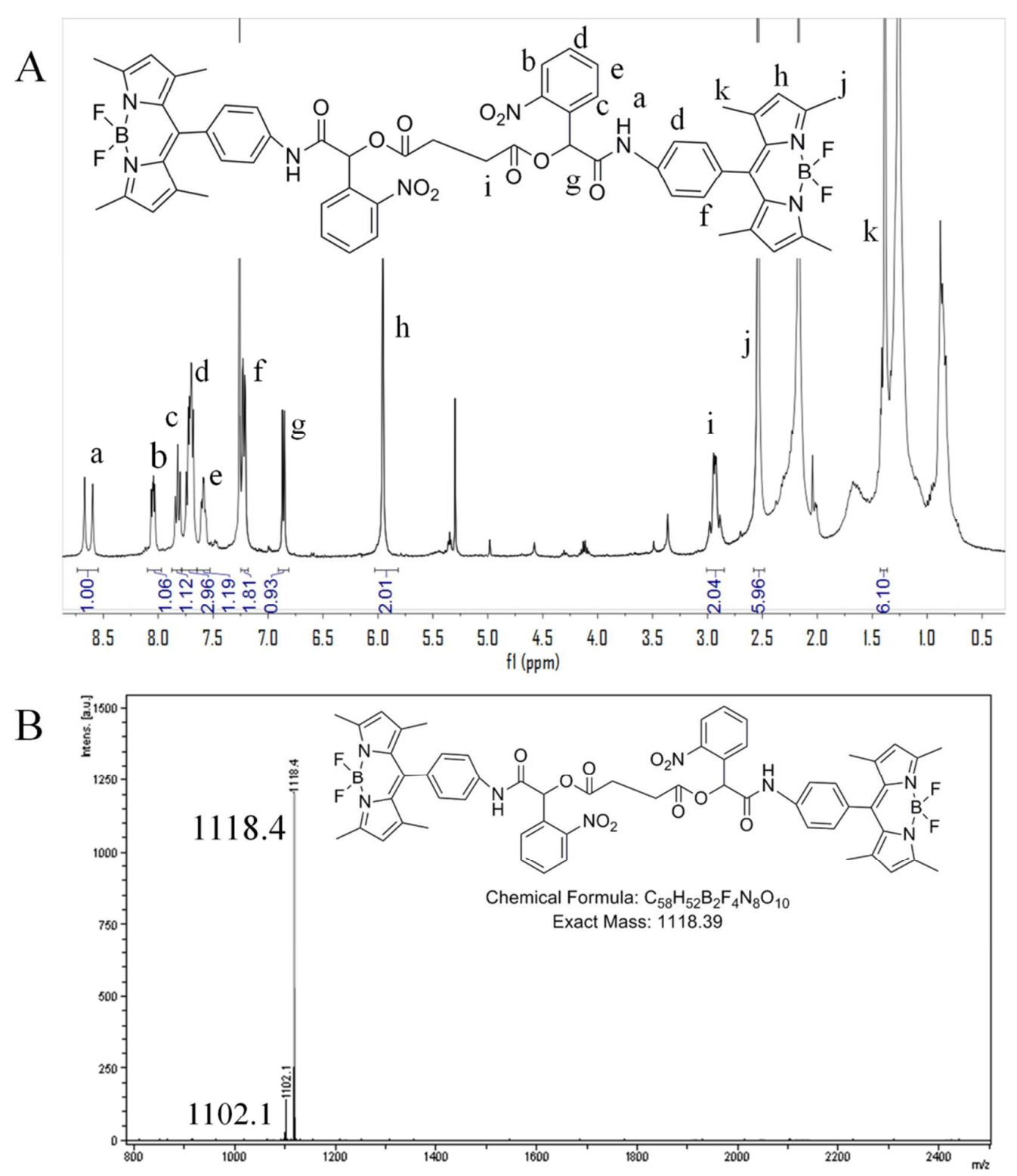

Figure S2. (A) ${ }^{1} \mathrm{H}$ NMR characterization and (B) MALDI-TOF MS spectrum of C4NBDP. 

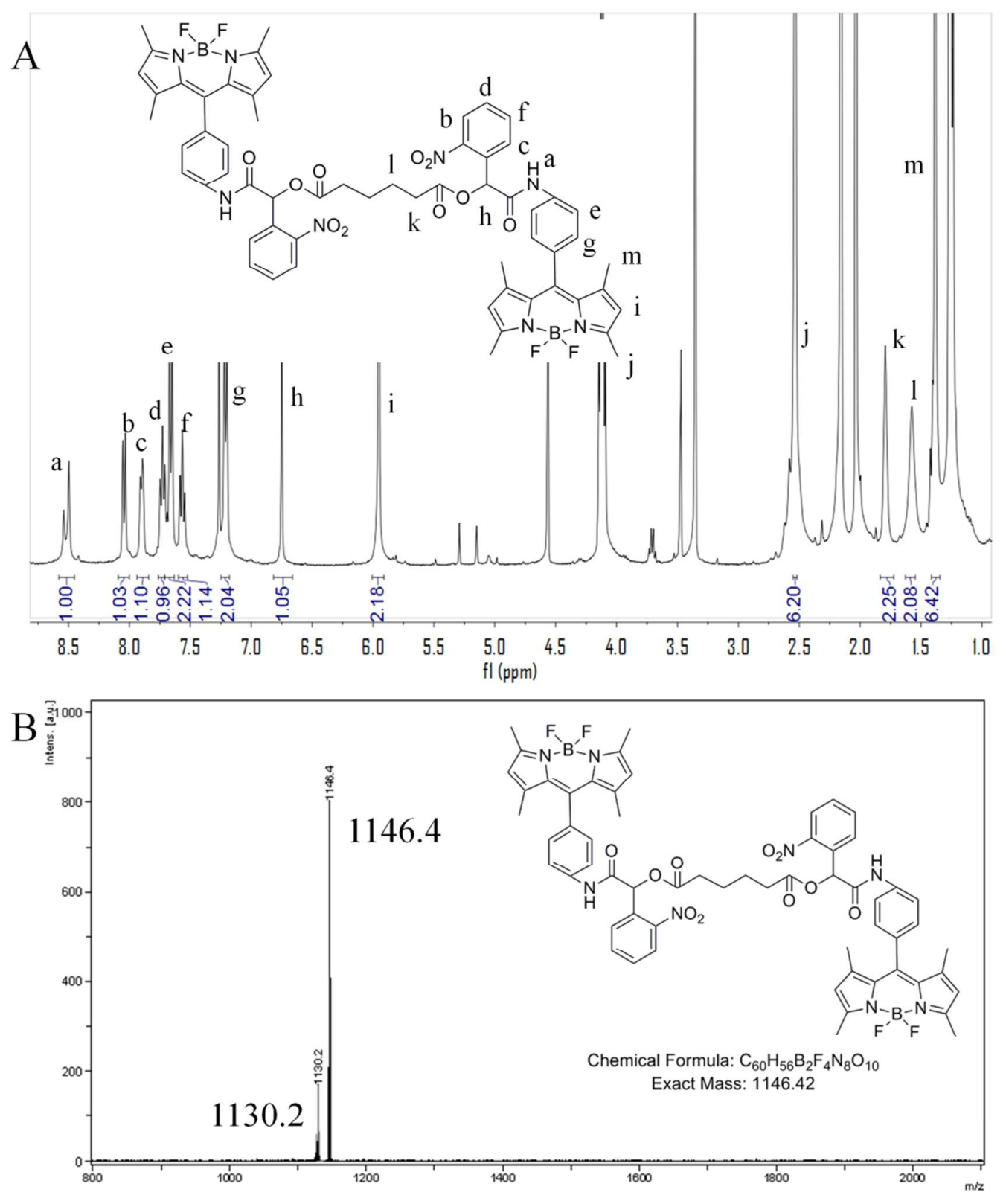

Figure S3. (A) ${ }^{1} \mathrm{H}$ NMR characterization and (B) MALDI-TOF MS spectrum of C6NBDP. 

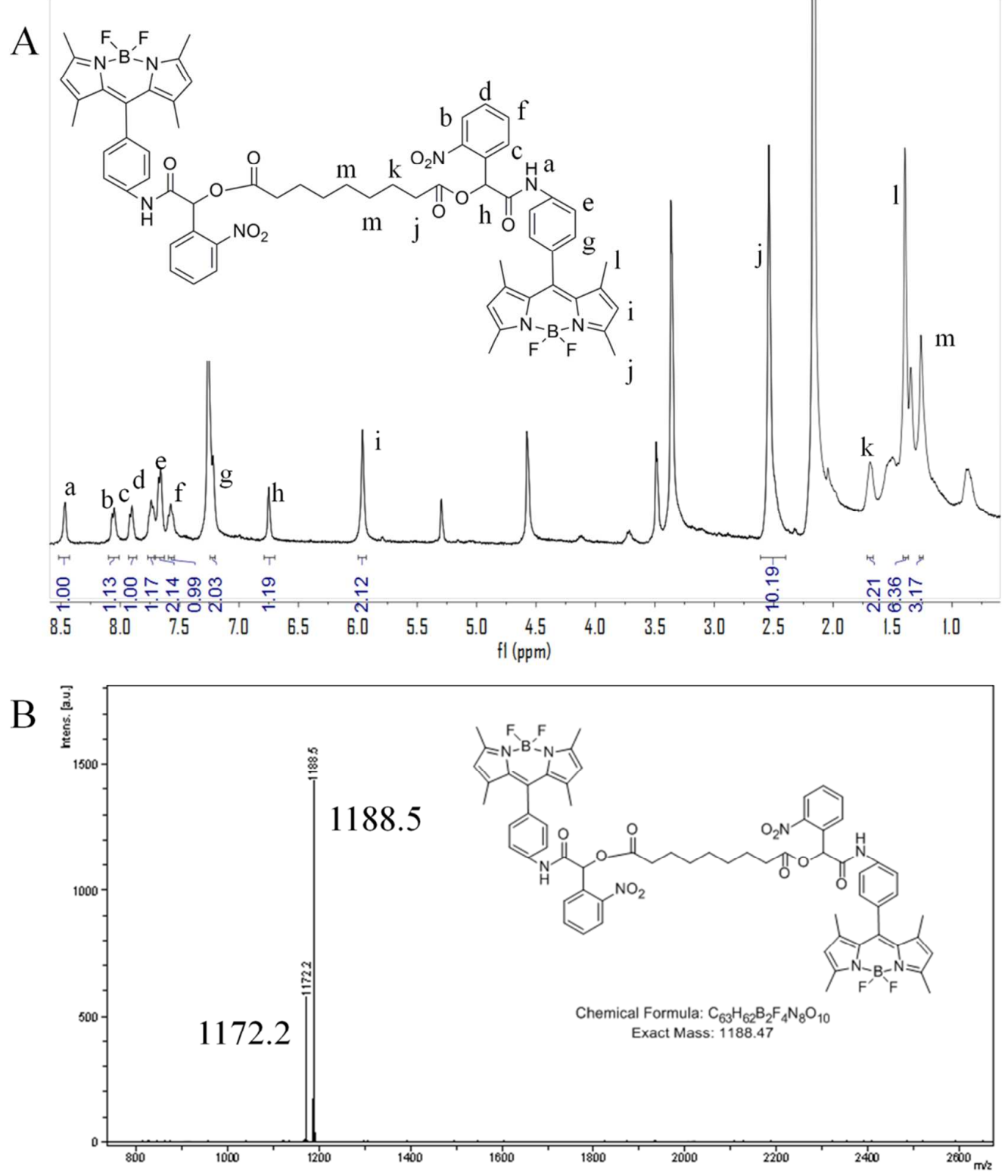

Figure S4. (A) ${ }^{1} \mathrm{H}$ NMR characterization and (B) MALDI-TOF MS spectrum of C9NBDP. 


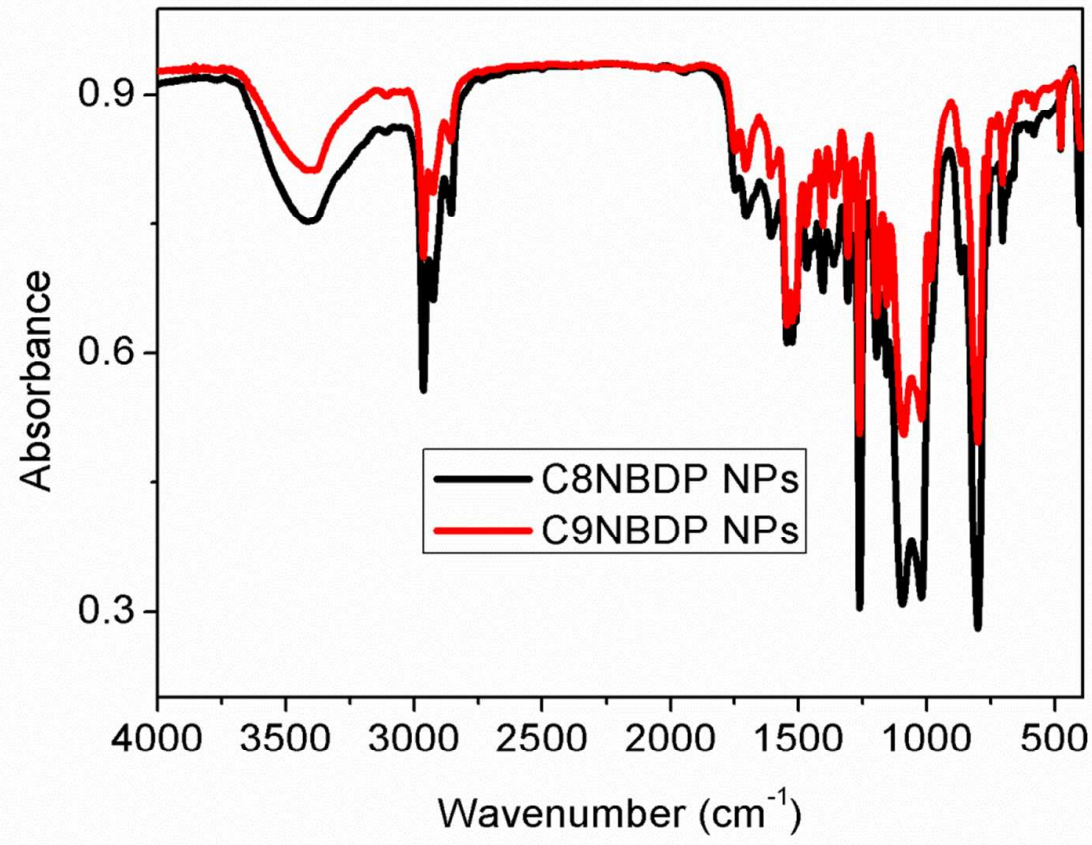

Figure S5. FT-IR spectra of C8NBDP NPs and C9NBDP NPs freeze-dried.
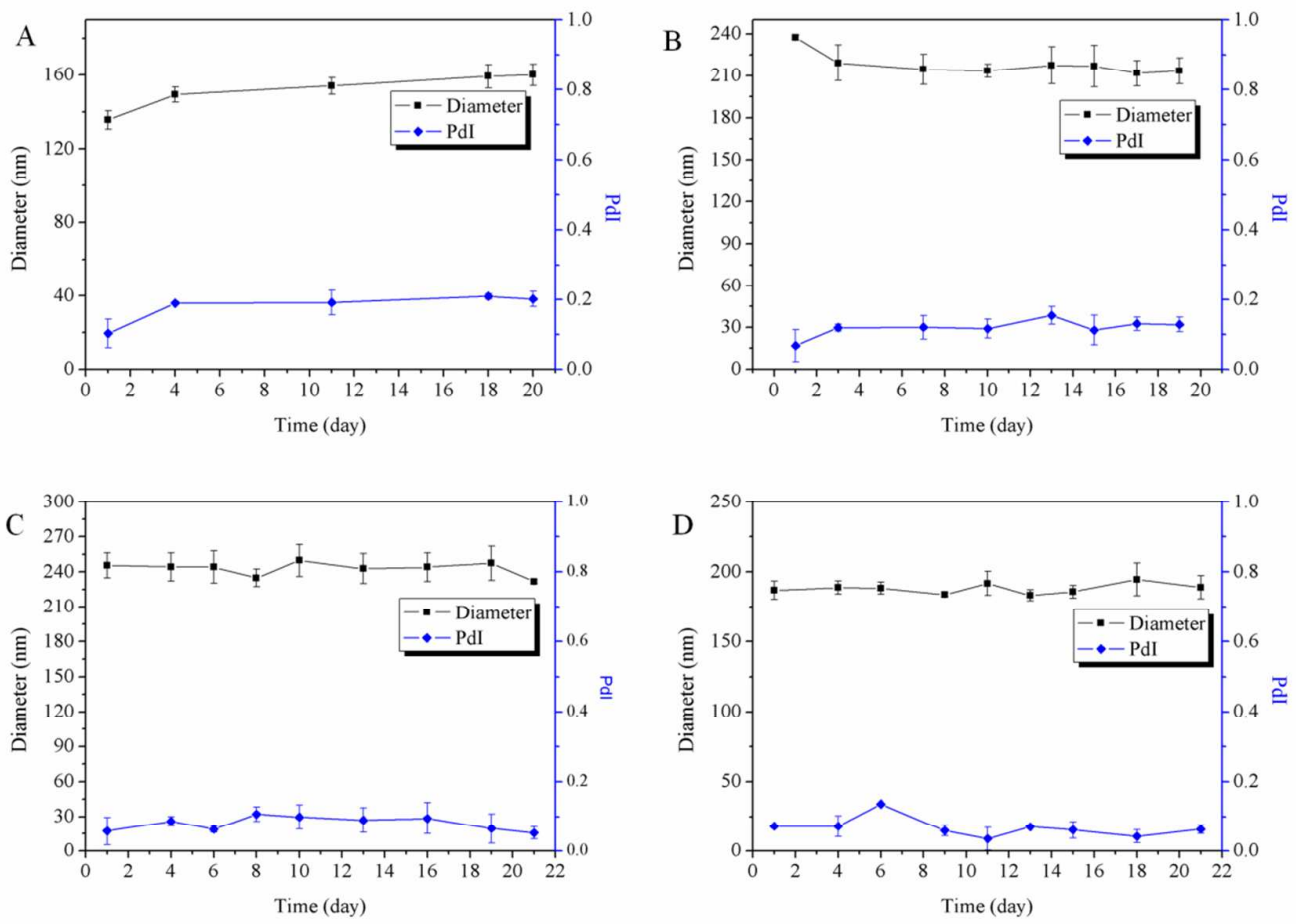

Figure S6. The diameter and PdI of (A) C4NBDP NPs, (B) C6NBDP NPs, (C) C8NBDP NPs and (D) C9NBDP NPs in twenty days measured by DLS. 


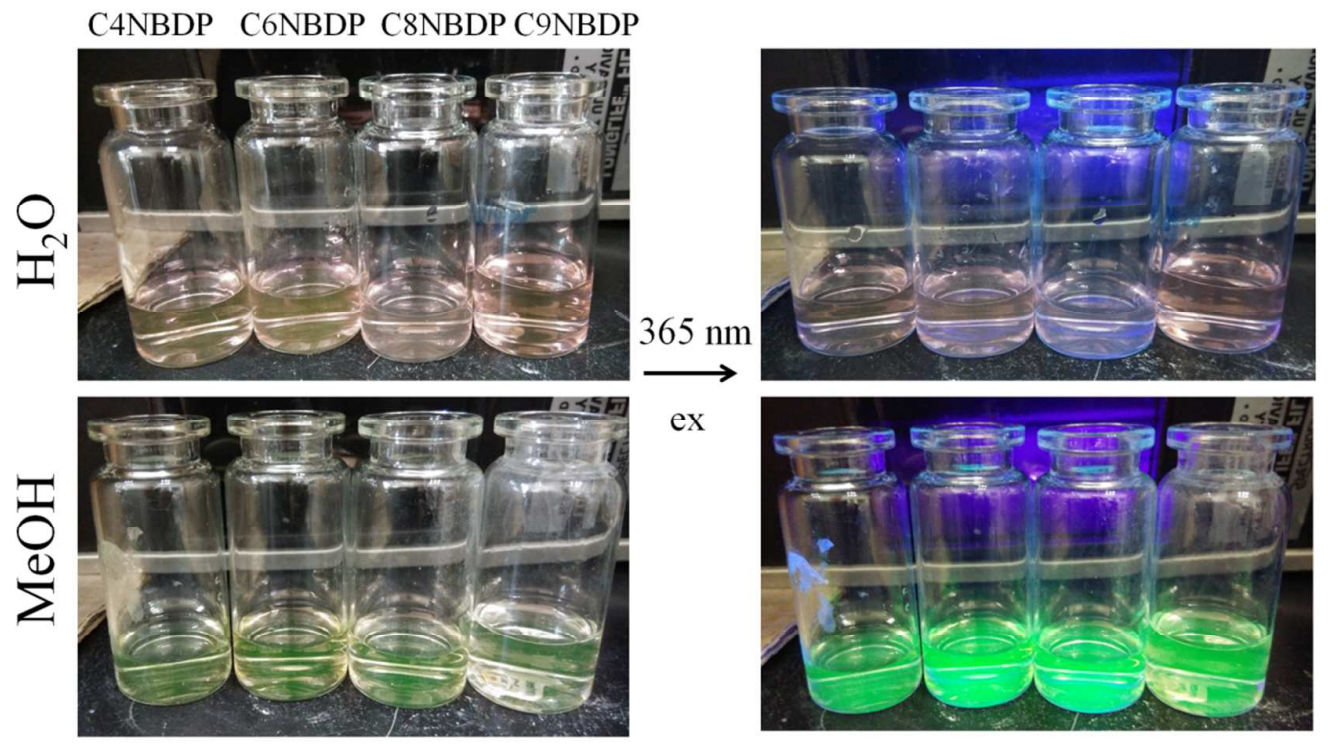

Figure S7. The photos of CxNBDP NPs in water and CxNBDP in MeOH under 365-nm light irradiation.

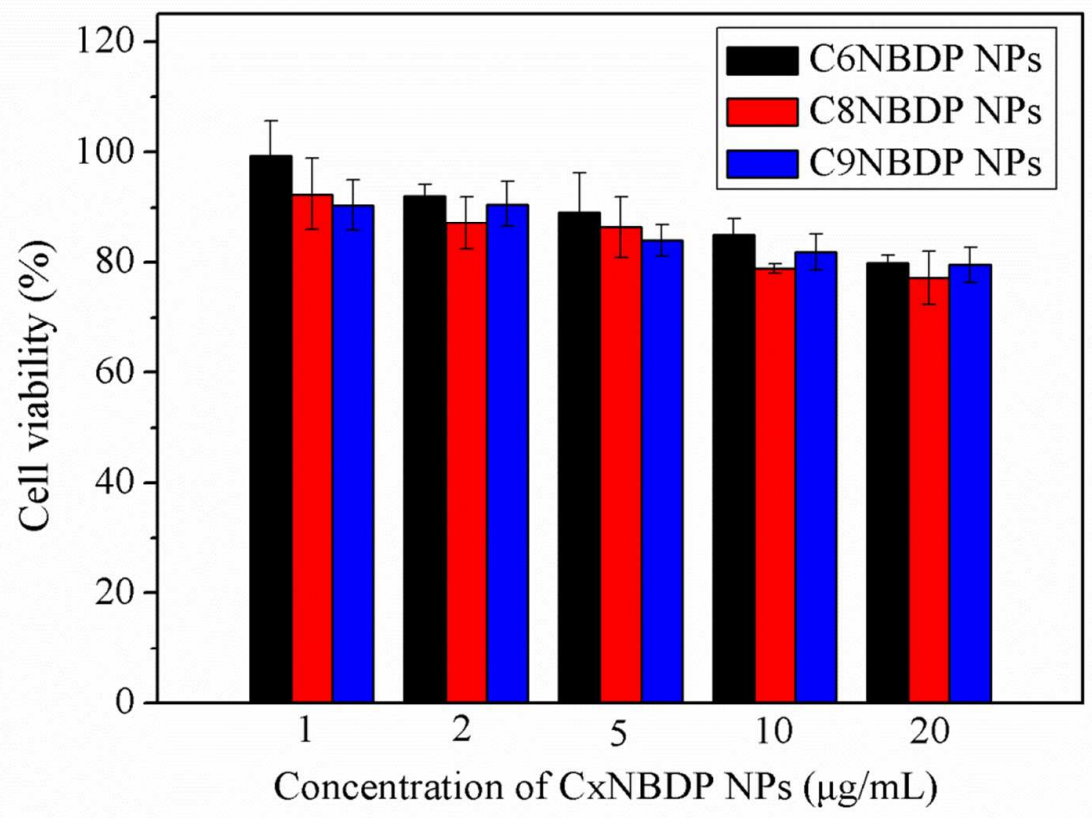

Figure S8. Cell viability of HeLa cells incubated with CxNBDP NPs 


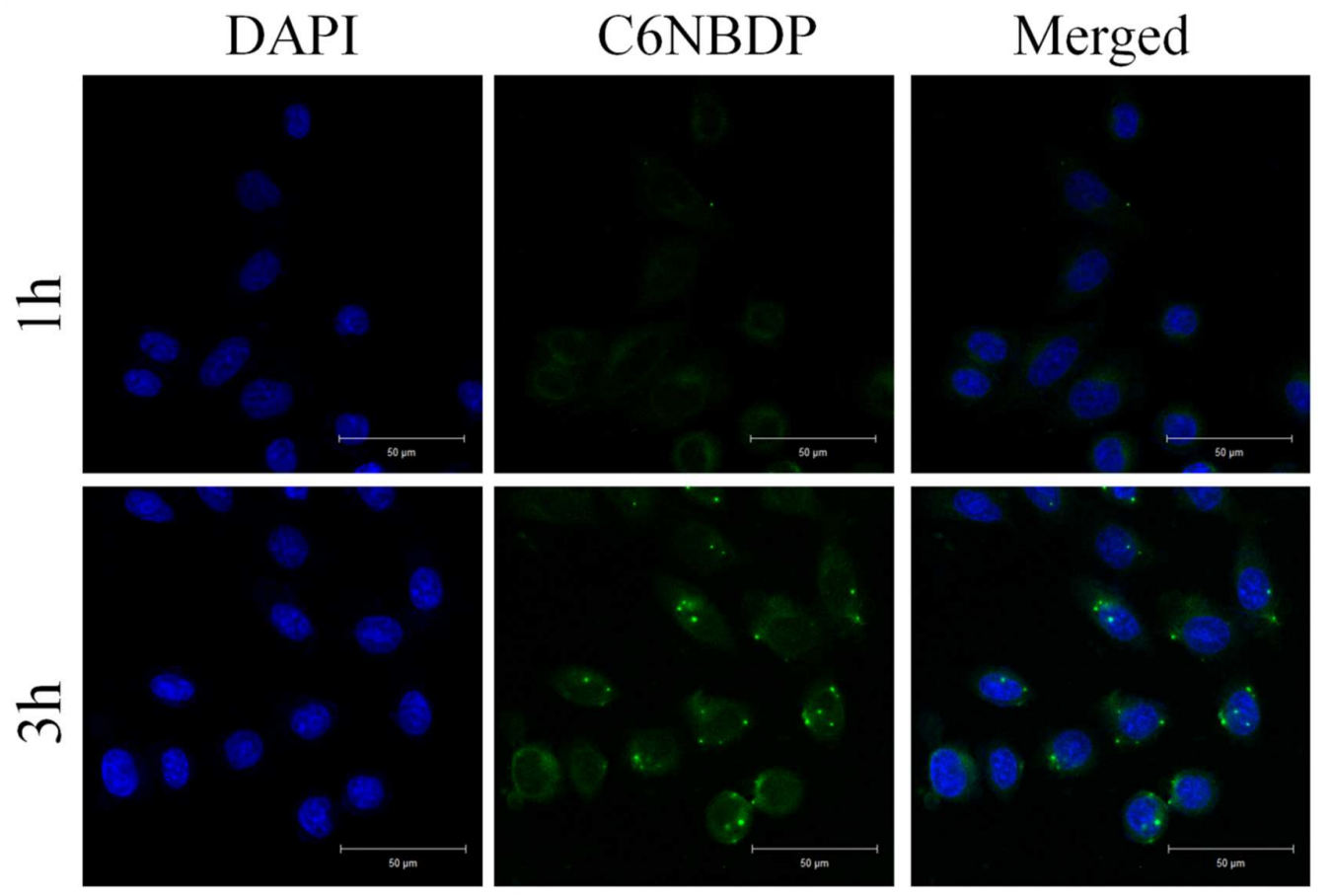

Figure S9. Respresentative CLSM images of HeLa cells incubated with C6NBDP

NPs for $1 \mathrm{~h}$ and $3 \mathrm{~h}$. 


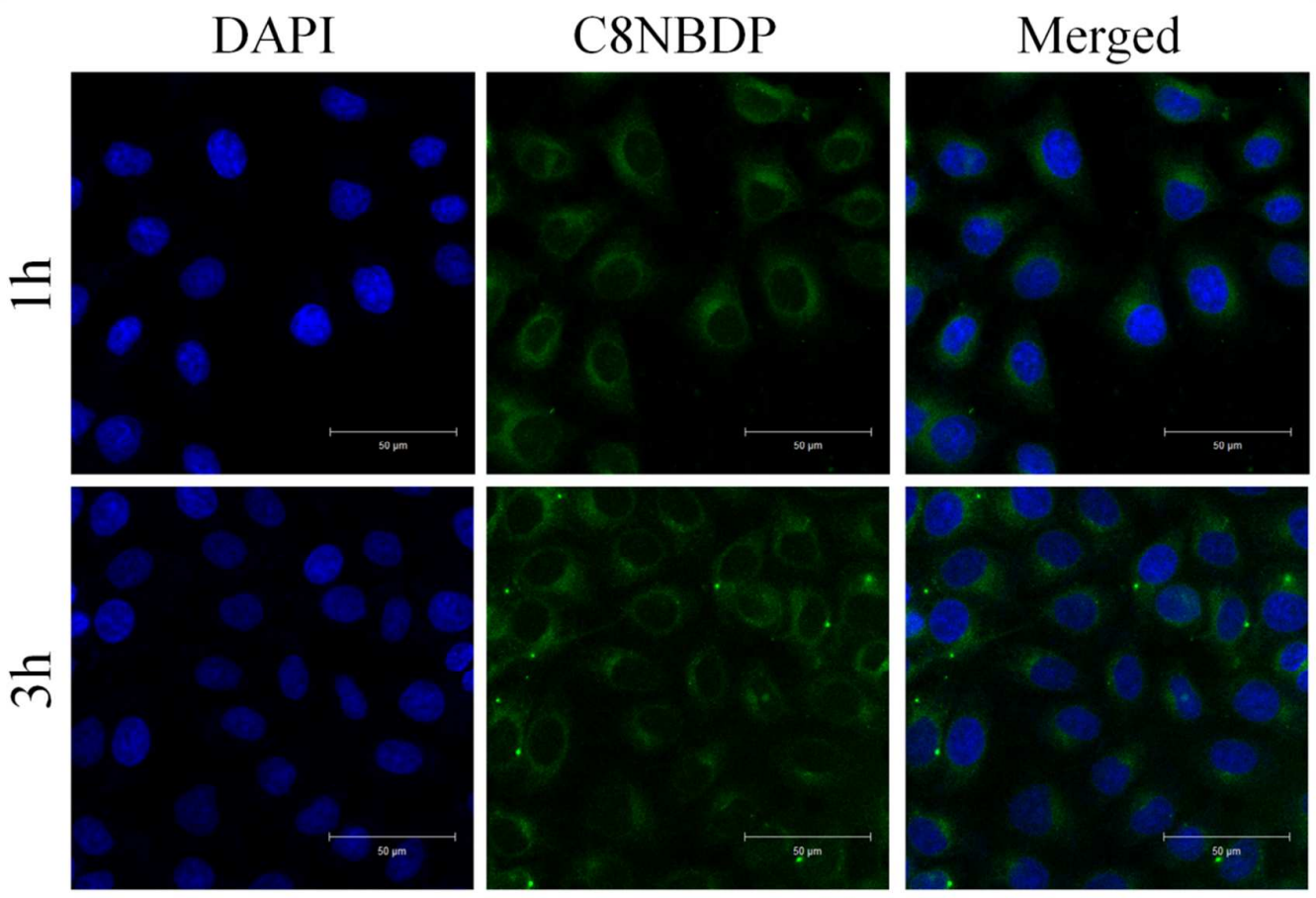

Figure S10. Representative CLSM images of HeLa cells incubated with C8NBDP

NPs for $1 \mathrm{~h}$ and $3 \mathrm{~h}$.
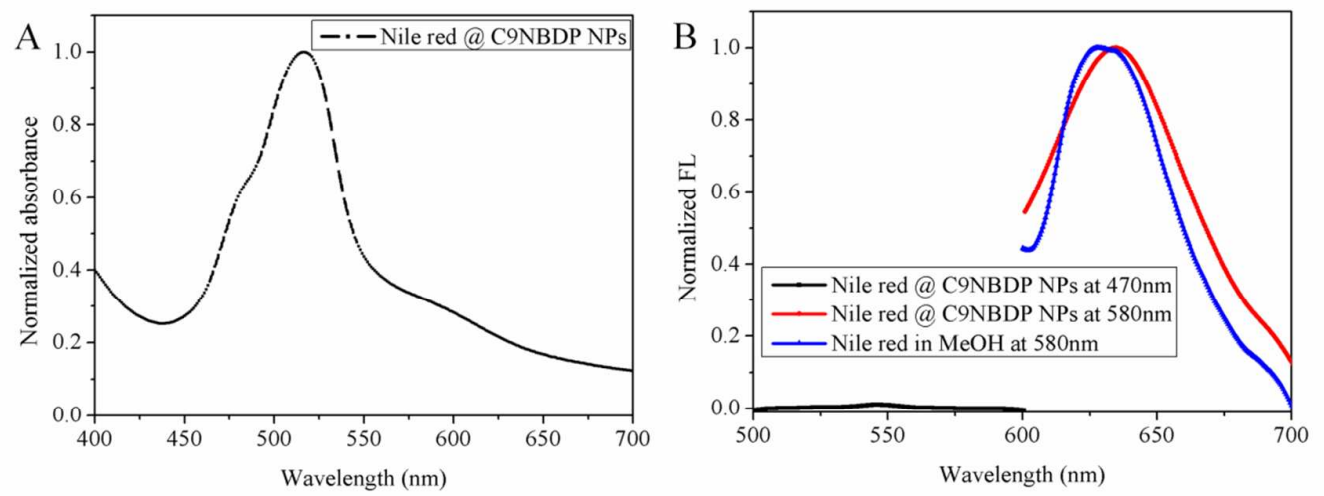

Figure S11. (A) UV-vis absorption and (B) fluorescence spectra of Nile red@C9NBDP NPs.

Table S1. Zeta potential of CxNBDP NPs

\begin{tabular}{ll}
\hline Nanoparticles & Zeta potential \\
\hline C6NBDP & $-30.1 \pm 1.2 \mathrm{mV}$ \\
C8NBDP & $-29.5 \pm 1.6 \mathrm{mV}$ \\
C9NBDP & $-28.3 \pm 0.2 \mathrm{mV}$
\end{tabular}

